\title{
Set size and ensemble perception of numerical value
}

\author{
Kassandra R. Lee ${ }^{1}$ (D) $\cdot$ Taylor D. Dague $^{2} \cdot$ Kenith V. Sobel $^{3} \cdot$ Nickolas J. Paternoster $^{4} \cdot$ Amrita M. Puri $^{3}$
}

Accepted: 29 October 2020 / Published online: 3 January 2021

(C) The Psychonomic Society, Inc. 2021

\begin{abstract}
There is a growing body of research on ensemble perception, or our ability to form ensemble representations based on perceptual features for stimuli of varying levels of complexity, and more recently, on ensemble cognition, which refers to our ability to perceive higher-level properties of stimuli such as facial attractiveness or gaze direction. Less is known about our ability to form ensemble representations based on more abstract properties such as the semantic meaning associated with items in a scene. Previous work examining whether the meaning associated with digits can be incorporated into summary statistical representations suggests that numerical information from digit ensembles can be extracted rapidly, and likely using a parallel processing mechanism. Here, we further investigate whether participants can accurately generate summary representations of numerical value from digit sets and explore the effect of set size on their ability to do so, by comparing psychometric functions based on a numerical averaging task in which set size varied. Steeper slopes for ten- and seven-item compared to five-item digit sets provide evidence that displays with more digits yield more reliable discrimination between larger and smaller numerical averages. Additionally, consistent with previous reports, we observed a response bias such that participants were more likely to report that the numerical average was "greater than 5" for larger compared to smaller sets. Overall, our results contribute to evidence that ensemble representations for semantic attributes may be carried out via similar mechanisms as those reported for perceptual features.
\end{abstract}

Keywords Ensemble perception $\cdot$ Semantic information $\cdot$ Average estimates $\cdot$ Summary representations

Ensemble perception is defined as an ability to extract summary information from groups of stimuli and is made possible because of our visual system's sensitivity to patterns of similarity across sets of items in a scene (Whitney \& Yamanashi Leib, 2018). This ability to generate ensemble percepts has been demonstrated for stimuli containing low-level perceptual features such as motion direction, orientation, and object size, among others (Alvarez \& Oliva, 2008; Alvarez \& Oliva, 2009; Dakin \& Watt, 1997; Oriet \& Corbett, 2008; Parkes et al., 2001; Watamaniuk et al., 1989; Williams \& Sekuler,

Amrita M. Puri

apuri@uca.edu

1 Department of Psychology and Center for Integrative Neuroscience, University of Nevada Reno, Reno, NV, USA

2 Department of Biology, University of Central Arkansas, Conway, AR, USA

3 Department of Psychology and Counseling, University of Central Arkansas, Conway, AR 72035, USA

4 Department of Computer Science, University of Central Arkansas, Conway, AR, USA
1984). The formation of higher-level gist representations, also referred to as ensemble cognition, is somewhat less studied (Whitney \& Yamanashi Leib, 2018), although it has been shown for properties of complex stimuli such as attractiveness of faces, animacy of objects, and direction of biological motion in crowds (Anderson et al., 1973; Yamanashi Leib et al., 2016; Sweeny et al., 2013).

While the studies mentioned above demonstrate ensemble cognition for higher-level features, less is known about whether the abstract meanings and semantic associations of items in a scene can be similarly extracted to form an ensemble representation. Brezis et al. (2018) showed that average estimation precision increases when items within temporally presented digit sequences are displayed for longer durations, and supported this finding with a population coding model. Two previous studies that examined whether the semantic meaning associated with digits can be incorporated into summary statistical representations, and whether this process relies on mechanisms that support ensemble coding in a visual context, suggest that people are able to form ensemble representations for numerical meaning (Lee et al., 2018; Van Opstal et al., 2011). In addition, earlier work found that numerical meaning assisted in comparisons of digit groups and provided evidence 
that extraction of this abstract information occurs more quickly than that of other, low-level stimuli for classification tasks (Corbett et al., 2006). Such evidence for ensemble coding of semantic information supports that extraction of summary information can occur at multiple levels of complexity, beyond perceptual features and even higher-level attributes that are thought to drive attention and behavior, such as emotion or other complex features from a crowd of faces (Haberman \& Whitney, 2007; Haberman \& Whitney, 2009).

In one seminal study investigating the nature of ensemble perception (Williams \& Sekular 1984), participants were asked to report the direction of coherent motion when many different motion directions were combined. Although each element in the random dot kinematograms moved independently of the other elements, participants were able to detect a coherent pattern of motion by pooling information across individual items. Although some studies have demonstrated that attention can bias averages (Chong \& Treisman, 2005b; De Fockert \& Marchant, 2008) and that divided attention can affect both accuracy and efficiency (Brand et al., 2012; Dakin et al., 2009; Huang 2015), others have shown that recognition of single items in a display is not necessary for ensemble coding to occur. In experiments in which attention was diminished to the stimulus set of interest, or to individual stimuli within a set, participants still accurately reported the average spatial position (Alvarez \& Oliva, 2008), circle size (Chong \& Treisman 2005a), and orientation (Alvarez \& Oliva, 2009) of display elements. While these studies specifically investigated low-level stimulus features, ensemble perception under conditions of limited attention has also been shown for faces, which contain higher-level features by virtue of being processed holistically (Tanaka \& Farah, 2003). For example, when faces were presented too briefly for participants to register the individual faces, they could nevertheless accurately report the average facial expression of the ensembles (Haberman et al., 2009).

The fact that processing each item individually is not required in order to extract ensemble information suggests at least two things. First, that ensemble perception represents an efficient form of processing where we trade consciously processing details from individual elements (or at least details that are unreportable) for an efficient summary statistical representation of a scene, providing us with an informative percept in just a brief glance (Block, 2011; Cohen et al., 2016; McClelland \& Bayne 2016); and second, that ensemble representations at different levels of processing likely depend on extraction of information from multiple items in parallel as opposed to serial extraction from one item at a time (e.g., Alvarez, 2011; Lee et al., 2018; Van Opstal et al., 2011). Lee et al. (2018) found evidence that ensemble information based on the numerical values associated with a digit set may be extracted via a parallel processing mechanism, with reaction time patterns for the numerical ensemble task resembling those observed for a parallel search task. The finding by Van Opstal et al. (2011) and Lee et al. (2018) that people can extract ensemble information from digits provides initial evidence that ensemble representations are formed for more abstract stimulus attributes.

Van Opstal et al. (2011) demonstrated that the semantic meanings of digits can be incorporated into an ensemble representation by asking participants to extract either the mean or the sum of target digits, and to compare it with a reference value, and also showed that digit arrays presented subliminally influenced ensemble estimates. Lee et al. (2018) extended this work by utilizing a larger range of set sizes and comparing the reaction time patterns for estimating the numerical mean of displays to those generated by searching for a specific digit within the display. They found that digit numerical averages are likely achieved by a parallel rather than serial process. And in fact, they found that reaction times decreased, and accuracy increased as the number of display items increased, indicating a potential benefit of larger displays.

While there is some variability and debate regarding the number of stimuli that can be integrated into an ensemble representation (Whitney \& Yamanashi Leib, 2018), many studies have shown that participants gather low-level information from three to five items in a display (Im \& Halberda, 2013; Solomon, 2010) and up to eight objects for higherlevel percepts (Haberman \& Whitney 2010; Sweeny et al., 2013). A study by Robitaille and Harris (2011) found an increase in performance for larger vs. smaller sets of oval and line stimuli, and Sweeny and Whitney (2014) found that additional faces in a display may increase the efficiency of generating the ensemble statistic for gaze direction. It has been demonstrated that more items in a display can lead to enhanced performance on ensemble tasks, likely related to the fact that the number of items sampled also grows with set size. More specifically, it has been approximated that observers integrate across the square root of the number of items in a set (Whitney \& Yamanashi Leib, 2018). It is unclear if this same benefit of larger sets extends to the semantic meaning associated with numerical stimulus sets.

Here, we extend the literature on ensemble cognition by further investigating ensemble perception of semantic associations. Specifically, we aim to determine how the ability to estimate the numerical average of a digit ensemble is affected by the inclusion of additional items in the display. The current study builds on limited previous work on ensemble perception of numerical information (Corbett et al., 2006; Lee et al., 2018; Van Opstal et al., 2011) to further our understanding of how semantic information is extracted from ensembles and provide insight into the underlying mental mechanisms. We asked participants to estimate the numerical average of briefly displayed digit arrays with true averages that varied in their distance from five, and analyzed psychometric functions to 
assess the effect of additional digits in the display on performance of the numerical averaging task.

\section{Method}

\section{Participants}

We obtained permission to carry out the experiment from our university's Institutional Review Board prior to collecting data and treated all participants in accordance with the ethical guidelines stipulated by the American Psychological Association (2017). A total of 19 undergraduate students at a public university in the midwestern U.S. volunteered to participate in exchange for class credit. Data from three participants were excluded from analysis: two due to extremely long RTs (more than 5 standard deviations greater than the mean RT across participants), and a third because of overall accuracy near chance $(64 \%)$. The remaining 16 participants (four male, 12 female) were between the ages of 18 and $22(\mathrm{M}=$ $20.19, \mathrm{SD}=1.28$ ) and had normal or corrected-to-normal vision. This sample size was based on our previous work comparing the RT pattern for a digit ensemble task to that for a digit visual search task (Lee et al., 2018). The current ensemble experiment is similar to the previous task, for which a power analysis with a predicted medium effect size estimated the need for ten participants. In the earlier as well as the current study, we reported data from 16 participants, which is similar to sample sizes reported for comparable tasks in the visual search literature as well as in earlier studies of ensemble perception (Alvarez \& Oliva, 2008; Corbett et al., 2006; Haberman \& Whitney, 2007).

\section{Stimuli}

Trials began with a screen displaying a fixation cross for 500 $\mathrm{ms}$, followed by a digit display that remained visible for 1500 ms. Digits were presented in white text on a black background in a circular array and were constructed from line segments similar to that of a digital clock-face, as depicted in Fig. 1. At a viewing distance of about $60 \mathrm{~cm}$, each digit was $0.92^{\circ}$ degrees of visual angle wide by $1.8^{\circ}$ tall. Digits were arranged on an imaginary circle with a radius of $5.9^{\circ}$ centered on a fixation cross spanning $1.0^{\circ}$ on each side. Displays consisted of a fixation point at the screen's center, surrounded by five, seven, or ten digits, ranging from 1 to 9 , but excluding 5 so as not to interfere with participants' ability to determine the average. The numerical distance from five of the true numerical averages of the displays varied such that for each set size, the numerical average could take on a range of values between three and seven (means for five- and ten-item displays: 3.0, 3.8, 4.6, 5.4, 6.2, 7.0; means for seven-item displays: 3.00 , $3.86,4.57,5.43,6.14,7.0)$.

\section{Procedure}

The ensemble task program was written in Xojo Basic and presented on a Mac computer connected to a built-in iMacdisplay with a screen resolution of 1920 x 1080 pixels. Participants performed an ensemble task in which they were instructed to estimate whether the average of each set of digits was less than or greater than five. Participants were instructed to press the " $z$ " key to indicate the mean was less than five, or the " " key to indicate that the mean was greater than five. Participants were asked to make their judgements via key presses as quickly and accurately as possible. A session included 12 practice trials and 16 trials for each of the six true numerical averages for each of the three display sizes for a total of 288 experimental trials.

\section{Analysis}

Participants' responses (greater than or less than 5) and reaction times (RTs) for all trials across display sizes were recorded. The experimental session for one participant was interrupted due to technical difficulties; this partial data set contained half the trials but the same distribution of set sizes and true averages as the full set of trials, and was included in the analyses. We compared performance across set sizes by fitting the proportion of "greater-than-five" responses given by individual participants for each true numerical average with a logistic function, $y=1 /\left(1+\mathrm{e}^{-\alpha(\mathrm{x}-\beta)}\right)$, in which the coefficients $\alpha$ and $\beta$ represent the slope and the midpoint ( $50 \%$ " $>5$ " responses), respectively. We used the slope and midpoint values, as well as RTs, for each participant in the analyses described below.

\section{Results}

A one-way ANOVA with set size (i.e., five, seven, or ten display items) as a within-subject factor conducted on the slope values revealed a main effect of set size on performance, $F(2,30)=4.47, p=.02, \eta_{\mathrm{p}}^{2}=.23$, such that slopes were steeper with larger set sizes (Fig. 2, panel a). Follow-up pairwise comparisons showed that this effect was due to significantly steeper slopes for the ten-item $(\mathrm{M}=2.15, \mathrm{SD}=$ $0.73)$ and seven-item $(\mathrm{M}=2.10, \mathrm{SD}=0.74)$ conditions compared to five-item $(\mathrm{M}=1.71, \mathrm{SD}=0.49)$ condition, $t(15)=$ $2.76, p=.015, d=.71$, and $t(15)=2.55, p=.022, d=.66$, respectively. Slopes were not significantly different across the seven- and ten-item conditions, $t(15)=-.26, p=.798, d=.07$.

Perhaps somewhat surprising given that psychometric functions were steeper in the ten- and seven-item conditions compared to the five-item condition, a one-way ANOVA with set size $(5 / 7 / 10)$ as a within-subject factor indicated that differences in accuracy across the five-item $(\mathrm{M}=82.02 \%, \mathrm{SD}=$ 


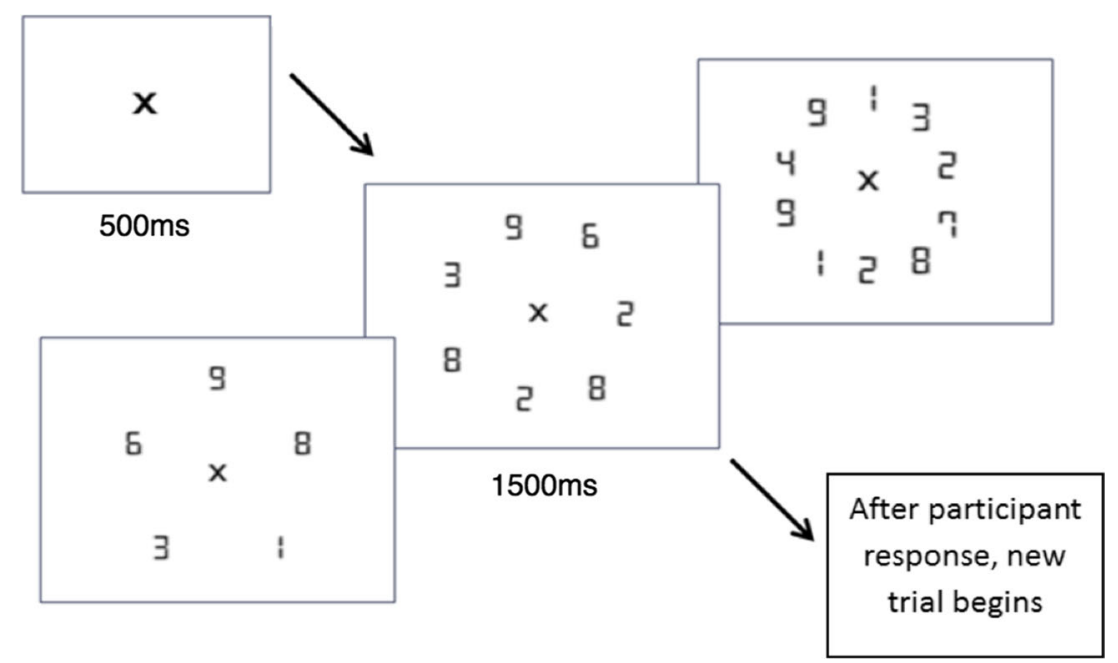

Fig. 1 Ensemble task procedure. Participants were instructed to estimate whether the average of a set of digits was greater than or less than 5 and respond by pressing the " $z$ " key for $<5$ and the " " key for $>5$. Sets could

4.07), seven-item $(\mathrm{M}=83.93 \%, \mathrm{SD}=5.79)$, and ten-item displays $(\mathrm{M}=82.22 \%, \mathrm{SD}=5.83)$ were not significant, $F(2$, $30)=1.70, p=0.20, \eta_{\mathrm{p}}^{2}=.10$.

Further examination of the psychometric functions revealed that larger set sizes led to a greater proportion of "> 5 " responses. We quantified this effect on the point of subjective equality (PSE), in this case the true numerical average that corresponds to $50 \%$ " $>5$ " responses, by subjecting the midpoint values of the psychometric functions for each set size for individual participants to a one-way ANOVA with set size $(5 / 7 / 10)$ as a within-subject factor. A main effect of set size, $F(2,30)=21.26, p<.0001, \eta_{\mathrm{p}}^{2}=.59$, was due to significant differences between the ten- $(\mathrm{M}=4.40, \mathrm{SD}=.37)$ and sevenitem $(\mathrm{M}=4.77, \mathrm{SD}=.29, t(15)=-4.37, p=.001, d=-1.09)$, seven- and five-item $(\mathrm{M}=5.09, \mathrm{SD}=.34, t(15)=-4.98, p=$ $.002, d=-.94)$, and ten- and five-item conditions $(t(15)=-$ $4.98, p<.001, d=-1.25)$. Figure 3 depicts the psychometric functions fit to the proportion of " $>5$ " responses for each true mean averaged across participants for each set size, showing contain 5, 7, or 10 digits. Digit displays were presented for $1500 \mathrm{~ms}$. The true numerical averages varied across six possible values for each set size, evenly distributed between 3 and 7 (but never 5)

steeper slopes, as well as a bias such that participants were more likely to report that the numerical average was "greater than 5", for larger compared to smaller sets.

Although our study was designed to examine psychometric functions, we also assessed the effect of set size on RTs to determine whether participants took longer to estimate means for larger sets. Although mean RTs for the seven- $(\mathrm{M}=1023$ $\mathrm{ms}, \mathrm{SD}=210.9)$ and ten- $(\mathrm{M}=999 \mathrm{~ms}, \mathrm{SD}=192.7)$ item trials were longer than for the five-item trials $(\mathrm{M}=983 \mathrm{~ms}, \mathrm{SD}=$ 47.9) (Fig. 2, panel b), a one-way ANOVA with set size (5/7/ $10)$ as a within-subject factor was not significant, $F(2,30)=$ $2.26, p=0.12, \eta_{\mathrm{p}}^{2}=.13$. Note also given the relatively small sets of integers used in the displays, it was possible to equate the means across the five- and ten-item displays (3.0, 3.8, 4.6, $5.4,6.2,7.0$; average distance from $5=1.20$ ), but for sevenitem displays, the means were, on average, slightly closer to five $(3.0,3.86,4.57,5.43,6.14,7.0$; average distance from $5=$ 1.19)), making the task slightly more difficult in the sevenitem condition.
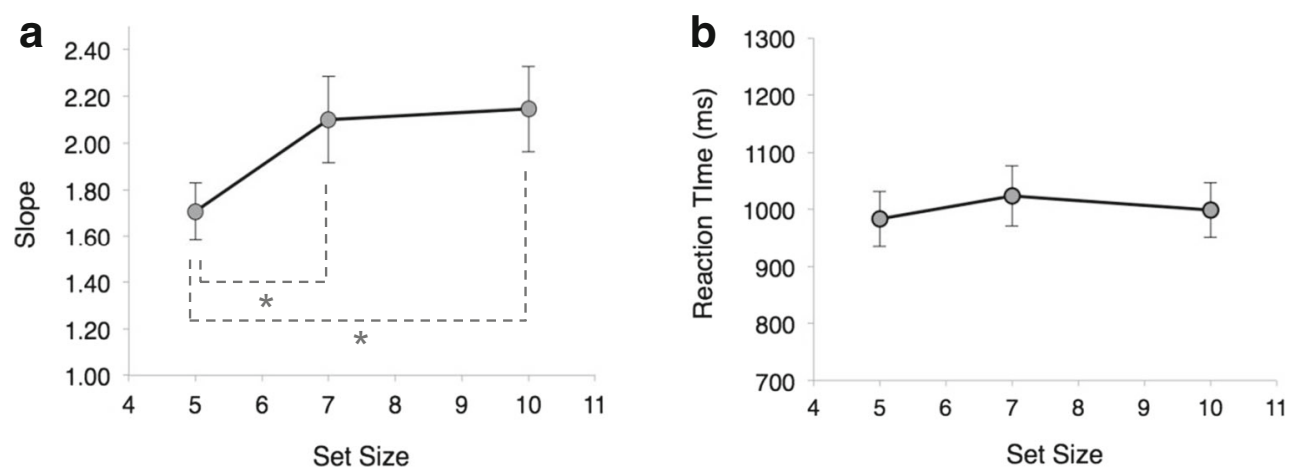

Fig. 2 a Average slopes of the psychometric functions across set size. Slopes were significantly steeper for the seven- and ten-item compared to the fiveitem displays $(* p<.05)$. b Reaction times as a function of set size. There was no significant effect of set size on RTs 


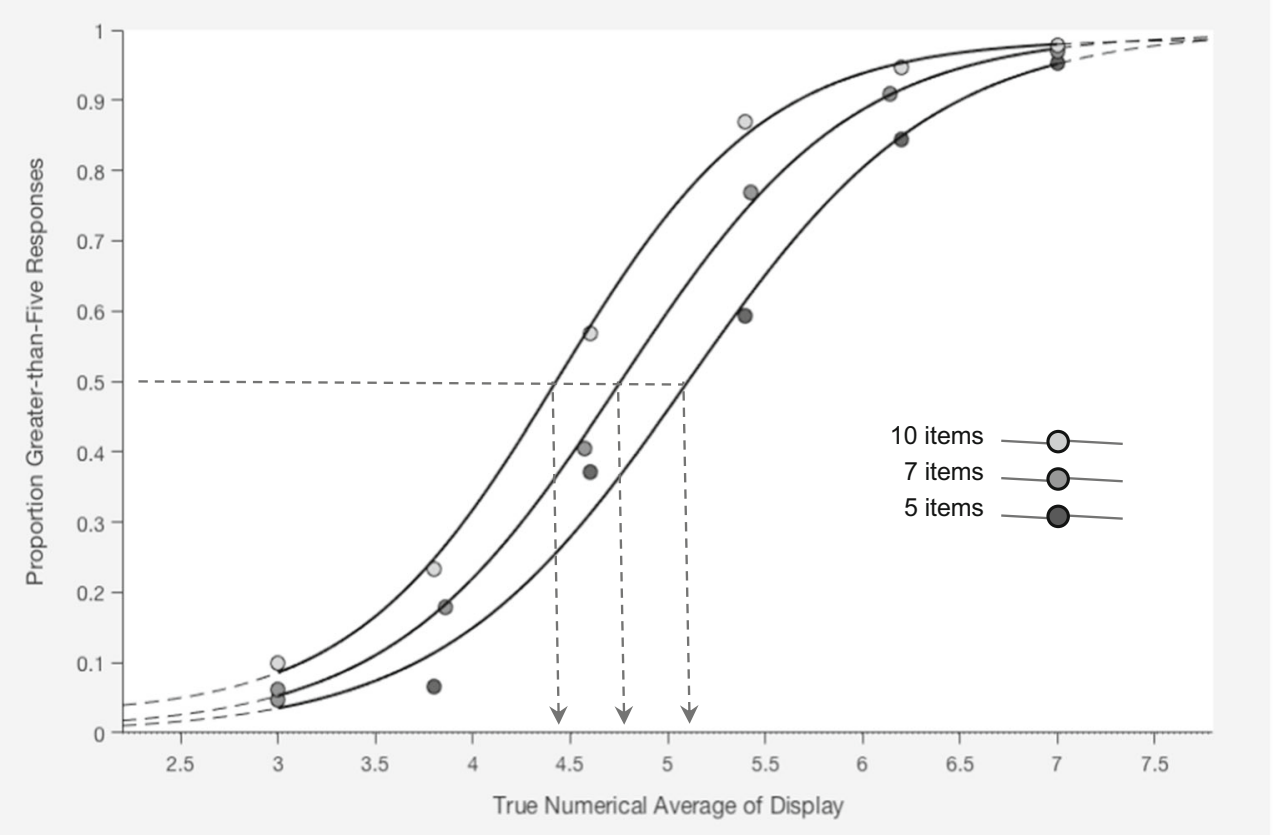

Fig. 3 Psychometric functions based on the proportion of "greater than five" responses for each true numerical average reflect set size effects on performance. Slopes were steeper for the seven- and ten-item compared to the five-item displays. Displays with a greater number of digits led to a

\section{Discussion}

Our goal in the current study was to better understand the ability to form ensemble representations based on semantic information from digit sets of varying sizes, and thus expand on a growing literature on ensemble cognition. Psychometric functions based on estimates of digit sets with different true numerical means were steeper for sets of ten compared to five digits; in addition, we observed a bias such that participants were more likely to report that the numerical average was "greater than 5", for displays with more items. This pattern of performance suggests that more digits in the display leads to more precise discrimination between larger and smaller numerical averages (indicated by steeper slopes), despite a shift in the PSE (or the perceived boundary between displays with greater-than-five and less-than-five averages). Although the lack of significant increases in RTs with larger sets suggests that participants did not require more time to generate these more precise estimates when more items were presented, we acknowledge that interpretation of this null effect is risky, particularly in light of our relatively small sample.

To address whether the lack of a significant effect of set size on RTs was simply due to a lack of power, we used a Bayesian approach to determine the posterior probability of the null and alternative hypothesis given our data. We restricted this follow up analysis to the ten- and five-item conditions, as longer RTs in the seven-item condition could be explained based on the true means for seven-item displays being closer to five (and thus more difficult to discriminate) compared to shift in the point of subjective equality (PSE) such that the true numerical average corresponding to $50 \%$ "greater than five" responses was smaller for larger set sizes (vertical arrows)

the five- and ten-item displays. A Bayesian paired samples $t$ test was conducted using JASP, a free software package for Bayesian analyses (JASP Team, 2020; Marsman \& Wagenmakers, 2017) to determine the relative predictive accuracy of the null hypothesis compared to a two-sided alternative hypothesis. Bayes factors calculated across a wide range of reasonable default scales for the Cauchy prior on effect size (Faulkenberry et al., 2020) indicated that our observed RT differences are approximately 3-4 times more likely under the null compared to the alternative hypothesis, further suggesting that RTs are not significantly affected by set size.

Whereas we observed both a bias towards perceiving larger sets as possessing greater averages, and more precise discrimination between displays with large and small numerical averages with increasing set size, we considered factors that may have contributed to these effects aside from additional numerical information. In our displays, because digits were white against a black background, and displays with greater-thanfive averages tended to have more line segments, the greaterthan-five displays were brighter overall compared to those with less-than-five averages. Because they contained more digits, the difference in brightness between the greater-thanfive displays and less-than-five displays was greater for the larger set sizes (on average, the difference in the number of line segments between $>5$ and $<5$ displays for the five-item displays was 3.21 compared to 5.63 for ten-item displays). While participants are unlikely to be consciously using a brightness cue, especially with individually presented stimuli 
rather than a simultaneous comparison, it is possible that the greater brightness differences in ten-item compared to fiveitem displays facilitated performance (in the sense of yielding more reliable estimates) for larger sets.

To address this potential confound, we controlled for brightness differences post-hoc by identifying and removing the subset of four trials for each mean that, based on the number of line segments in the digits, contributed most to the brightness difference for ten-item displays and that contributed least to the brightness differences for the five-item displays. For the remaining trials (12 out of the original 16 trials for each of the six true numerical averages per set size condition) despite the fact that brightness differences were now larger for the five-item compared to ten-item displays (difference of 5.39 vs. 3.06 line segments, respectively), we observed similar slope and RT trends as observed in the full data set, although with fewer trials the slope differences were no longer significant. On the other hand, RT differences for this subset of trials with opposite brightness patterns approached significance, with the ten-item displays leading to slightly longer RTs compared to five-items (ten-item: $\mathrm{M}=1018, \mathrm{SD}=207$, five-item: $\mathrm{M}=974, \mathrm{SD}=197, t(15)=2.06, p=.057, d=.52$ ) (Fig. 4, panel a).

Although the observed trend of steeper slopes and longer RTs for large sets could potentially reflect a speed-accuracy tradeoff, crucially, the fact that we observed a similar pattern of steeper slopes for ten-item sets even when larger brightness differences were associated with the five-item sets demonstrates that brightness differences are unlikely to explain the steeper slopes with larger displays that we observed in the full data set. Nonetheless, to further address whether longer RTs could have led to improved precision of the average estimates, we looked at the relationship between the difference in RTs and the difference in slopes between ten- and five-item displays across participants. A positive correlation between these differences such that the participants who took longer on the ten- vs. five-item displays also showed a larger increase in

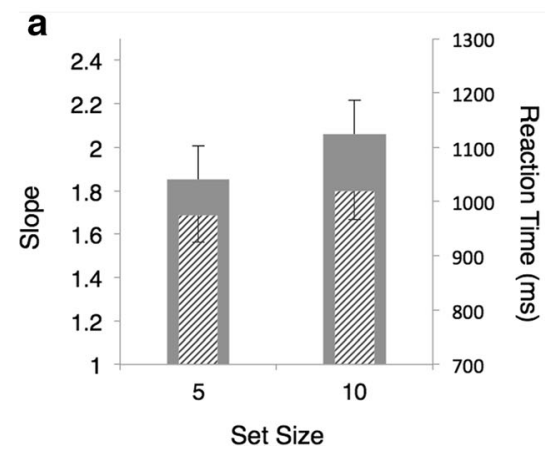

Fig. 4 a Average slopes of the psychometric functions (dark gray bars) and RTs (hatched bars) for the five- and ten-item displays after reversing the relative brightness differences between $>5$ and $<5$ displays across set sizes. The pattern of slope differences is the same as for the full data set, slopes between the ten- and five-item displays would suggest that longer RTs may play a role in the more reliable judgements for larger sets. However, the correlation was small and not significant, $r(14)=.09, p=.74$ (Fig. 4 , panel b). Thus, it is unlikely that the marginal increase in RTs for the ten-item sets explains the pattern of slope differences between ten- and fiveitem sets.

The finding that the number of items in a set does not have a negative effect on ensemble judgements has been demonstrated in several studies (Robitaille \& Harris, 2011; Sweeny \& Whitney, 2014; Utochkin \& Tiurina, 2014), although Marchant et al. (2013) suggest that these effects could be explained by a corresponding increase in the regularity of items in the larger sets. They showed that when larger set sizes were similarly heterogeneous, judgements became less rather than more accurate, at least for lower-level judgements of circle size, and interpret this to mean that ensemble representations may not pull from all items in parallel, but instead participants may employ a subset sampling strategy. In response to this claim, Utochkin and Tiurina (2014) analyzed the stimuli used by Marchant et al. (2013) and noted that their larger, irregular sets had larger variability in the size of circles (distance between the largest and smallest circles), which may have led to a decrease in performance. In equating true averages across set sizes, we were careful to sample from the whole range between 1 and 9 (except 5) rather than, for example, simply doubling our digit selection from the fiveitem condition to create the ten-item displays. We also avoided the use of more than three instances of any individual digit in a display in an attempt to limit homogeneity in larger displays. However, our use of single digits to generate sets corresponding to specific numerical averages restricted the degree to which we were able to control heterogeneity and variability. While the variance in the numerical value of digits was not significantly different across set sizes, our ten-item displays did include, on average, a greater number of repeated digits than five-item sets. It will be important in future work to

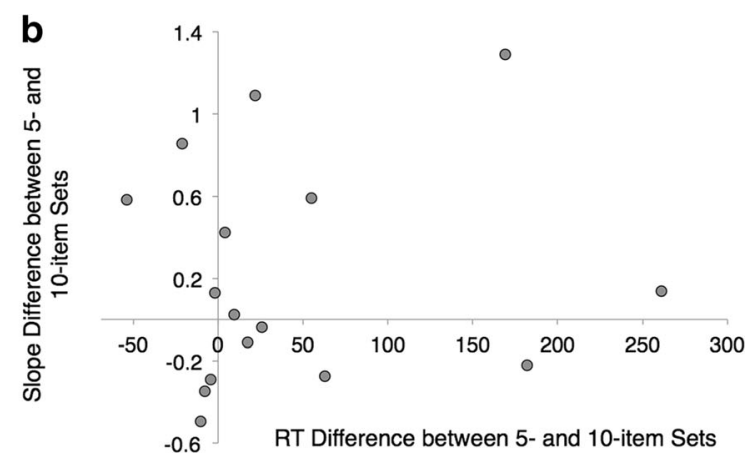

although not significant here. RTs were marginally longer for the ten-item compared to the five-item displays. $\mathbf{b}$ There was no relationship between the RT and slope differences between ten- and five-item sets across participants 
implement methods for controlling both variance and heterogeneity of digit displays across set sizes.

Our observation that ensemble judgements of numerical value, while shifted from the true mean, are not hindered by, or perhaps even improved (here, in terms of precision) with additional items, is consistent with the ensemble coding literature for lower-level stimuli and the idea that ensemble perception may arise via a parallel process (Ariely, 2001; Chong \& Treisman, 2003, Chong \& Treisman, 2005a). Previous work has shown that participants can extract information from digits presented rapidly, and used a population coding model to support the idea that this was accomplished without utilizing an arithmetic approach (Brezis et al., 2018). If observers were not calculating an arithmetic mean in our study, they were likely not processing each item serially (after all, if participants were able to calculate an average, we would expect much steeper slopes overall; here, for example, we observed that even for sets of just 5 digits, only $\sim 60 \%$ of responses were " $>5$ " when the true average was 5.4). Similarly, Van Opstal et al. (2011) reported that their participants likely were not calculating the exact sum or mean, but instead using an approximation strategy.

Although it is possible that participants were engaging a parallel processing mechanism to generate estimates of the numerical average from digit ensembles, our display duration of $1500 \mathrm{~ms}$ made it possible for observers to make several saccades during a trial, and greater display density with larger set sizes may have allowed observers to sample more items with each saccade. We chose this display duration based on those used in previous studies of ensemble perception of abstract digits (Van Opstal et al., 2011 \& Lee et al., 2018), and the notion that the time course of ensemble coding may vary depending on the type of stimulus or attribute (Whitney \& Yamanashi Leib, 2018). For example, ensemble coding for lower level stimulus characteristics such as size, orientation and motion direction has been demonstrated with display durations of 100-500 ms (Dakin et al., 2005; Chong et al., 2008; Solomon, 2010), whereas studies investigating ensemble cognition for complex aspects of stimuli such as facial attributes and gaze direction have employed durations closer to $1000 \mathrm{~ms}$ (Wolfe et al., 2015; Yamanashi Leib et al., 2014; Haberman \& Whitney, 2010). Nonetheless, future studies could manipulate display duration and analyze eye movements to test whether saccades during longer presentation durations play a role in ensemble processing of digits sets, or whether ensemble representations of numerical meaning are based on extraction of semantic information from multiple digits in parallel.

Though we did not predict it a priori, our most robust finding was a response bias towards "greater than five" estimates for larger set sizes. We ensured that the true averages across set sizes were balanced in their distance from 5 , so there was no difference in the true means driving this pattern. Instead, this is a trend that can be seen in tasks where participants are asked to estimate information for digits. Lee et al., (2018) reported greater accuracy for estimates of numerical averages with larger sets, specifically for displays with a true average of greater than five. Additionally, Smith and Price (2010) reported a similar bias such that as more numbers were included in a display, the magnitude of estimates of the numerical average increased even though participants were given longer to view the larger sets. One explanation for such a bias is that the set size may activate a magnitude judgement which is integrated into the estimation task. When using digits arrays in a statistical summary task, it is important to examine these biases as they may provide insight to the way participants are carrying out the task. For example, Van Opstal et al. (2011) found a bias for participants to overweigh the larger digits in a set when performing a summation task. This indicated that participants were not assigning equal weight to all values in a set, and therefore not completing an addition task as directed. For our averaging experiment, the observed bias could be due to overweighting of a subset of items and/or rather that larger sets may trigger a magnitude judgement that is incorporated into the estimate. Having an understanding of the way biases manifest in judgments of ensembles is of key importance in building future experiments that ensure we capture subjective judgements about perceived ensemble features of interest, as opposed to judgements influenced by extraneous features.

One form of perceptual bias that has been identified in the ensemble literature is referred to as the 'amplification effect', whereby individuals show a perceptual bias to preferentially weight salient items in a set as it increases in size (Kanaya, Hayashi, Whitney, 2018), rather than randomly weighting items when gathering ensemble information. Kanaya and colleagues found increased perceived means for both spatial and temporal ensemble tasks with increasing set size, suggesting saliency-based weighting. While the amplification effect does describe a form of bias seen during ensemble tasks, it may not explain our observed bias towards "greater than five" estimates for larger sets. For the amplification bias to account for our findings, some digits in our displays must be more salient than others, along the lines of stimuli with greater temporal frequencies and larger sizes in sets utilized by Kanaya et al., (2018). However, the most salient digits in our sets, if based on either low-level properties (e.g., fewer line segments) or semantic meaning, would have been the same across set sizes, and would consist of the digits on the "other side" of 5 , opposite of the true average. For example, if a given display has a true average greater than five, then the salient digit(s) would comprise the small subset of digits that are less than five, and vice versa. Such a source of bias should have canceled out across conditions in our design, thus we suspect that the shifts we observed in the PSE were independent of any amplification effect, but may be related to similar findings in previous studies utilizing digit stimuli (Smith \& Price, 2010; Van Opstal et al., 2011). 
We have shown that independent of contributions from low-level cues such as brightness or a set size bias, estimates of the numerical average of digit sets do not decrease as would be expected with brief displays if each item required individual processing, but rather increase in accuracy with additional digits in the set. This is consistent with previous ensemble perception literature and suggests we are able to gather numerical estimates in a manner comparable to that observed with lower-level stimulus properties, namely via parallel processing. Ensemble perception has been demonstrated across a number of perceptual and cognitive domains, such as orientation, object size, facial expression, gaze, and digit tasks, including averaging and classification (Alvarez \& Olivia, 2009; Corbett et al., 2006; Dakin \& Watt, 1997; Haberman et al., 2009; Lee et al., 2018; Parkes et al., 2001; Sweeny \& Whitney, 2014; Van Opstal et al., 2011). All of these domains naturally possess their own levels of processing complexity. For example, face processing is considered to be high-level, engaging a holistic processing approach (Tanaka \& Farah, 2003).

Perception of digits is also a complex process and involves interactions between the perceptual and semantic components of the alphanumeric character. Previous studies have tried to better understand this interaction between semantic and perceptual processing and have found evidence that semantic information affects judgements about perceptual attributes (Hansen et al., 2006; Lupyan et al., 2010; Puri \& Wojciulik, 2008) and can affect performance on behavioral tasks such as searching for letters or digits in an array (Lupyan, 2008; Sobel et al., 2015). Studies investigating the size congruity effect (SCE), which describes interference between physical and numerical size (semantic meaning) in digit comparison, have sparked a debate about whether these interact at an early perceptual processing stage or at a later, decision-related stage (Faulkenberry et al., 2016; Santens \& Verguts, 2011; Schwarz \& Heinze, 1998; Sobel et al., 2017; Walsh, 2003). Our finding that we can extract semantic information in an ensemble context may inform models of how perceptual and semantic information interact.

Because of the uncertainty surrounding how perceptual and semantic information influence each other, we do not know whether what we have observed about digital ensembles will generalize to other stimuli with associated semantic meanings. Haberman et al. (2015) conducted a thorough study of ensemble coding for different stimulus types to better understand the underlying mechanisms by examining individual differences for ensemble percepts. They found that ensemble representations of higher-level stimulus features were independent of lower-level ones, and that ensembles tended to cluster in their own domains of lower, mid-, and high-level processing information. This could mean that performance on a digit ensemble task such as the one here may not necessarily tell us anything about performance on lower-level features such as orientation, but may be able to tell us something about our ability to extract other statistical information from digits such as the sum or variance of digit sets. The relationship between these tasks and the process by which we complete them, however, is likely not straightforward. Future studies using digit ensembles could help us further understand the mechanisms underlying ensemble percepts. In addition, understanding the relationship between different types of ensemble tasks (i.e., high vs. low-level stimuli) could help us better parse out the relationship between processing of the lower-level perceptual features such as shape and physical size and higher-level semantic information possessed by digits.

Our findings provide support for the idea that semantic information can be extracted to form ensemble representations and suggest that additional items in a display do not hinder and may even improve the precision of high-level ensemble representations. These results contribute to our understanding of ensemble coding by providing additional evidence that it occurs for abstract stimulus associations such as numerical value. Our work thus paves the way for future research using digits or other complex stimuli with associated semantic features to better understand the scope of and mechanisms underlying ensemble coding.

Acknowledgements We would like to thank Dr. Thomas Faulkenberry for helpful discussion regarding the Bayesian analysis, and Christian Hopkins for assistance with fitting the psychometric functions.

Open practices statement This study was not preregistered. We are happy to make our data and materials available upon request.

\section{References}

Alvarez, G. A. (2011). Representing multiple objects as an ensemble enhances visual cognition. Trends in Cognitive Sciences, 15(3), $122-131$.

Alvarez, G. A., \& Oliva, A. (2008). The representation of simple ensemble visual features outside the focus of attention. Psychological Science, 19, 392-398. https://doi.org/10.1111/j.1467-9280.2008. 02098.x

Alvarez, G. A., \& Oliva, A. (2009). Spatial ensemble statistics are efficient codes that can be represented with reduced attention. Proceedings of the National Academy of Sciences of the United States of America, 106, 7345-7350. https://doi.org/10.1073/pnas. 0808981106

American Psychological Association. (2017). Ethical principles of psychologists and code of conduct. Retrieved from http://www.apa.org/ ethics/code

Anderson, N. H., Lindner, R., \& Lopes, L. L. (1973). Integration theory applied to judgments of group attractiveness. Journal of Personality and Social Psychology, 26(3), 400.

Ariely, D. (2001). Seeing sets: Representation by statistical properties. Psychological Science, 12, 157-162.

Block, N. (2011). Perceptual consciousness overflows cognitive access. Trends in Cognitive Sciences, 15(12), 567-575.

Brand, J., Oriet, C., \& Sykes Tottenham, L. (2012). Size and emotion averaging: Costs of dividing attention after all. Canadian Journal of 
Experimental Psychology/Revue canadienne de psychologie expérimentale, 66(1), 63.

Brezis, N., Bronfman, Z. Z., \& Usher, M. (2018). A perceptual-like population-coding mechanism of approximate numerical averaging. Neural Computation, 30(2), 428-446.

Chong, S. C., \& Treisman, A. (2003). Representation of statistical properties. Vision Research, 43, 393-404.

Chong, S. C., \& Treisman, A. (2005a). Statistical processing: Computing the average size in perceptual groups. Vision Research, 45(7), 891900.

Chong, S. C., \& Treisman, A. (2005b). Attentional spread in the statistical processing of visual displays. Perception \& Psychophysics, 67(1), $1-13$.

Chong, S. C., Joo, S. J., Emmmanouil, T. A., \& Treisman, A. (2008). Statistical processing: Not so implausible after all. Perception \& Psychophysics, 70(7), 1327-1334.

Cohen, M. A., Dennett, D. C., \& Kanwisher, N. (2016). What is the bandwidth of perceptual experience?. Trends in Cognitive Sciences, 20(5), 324-335.

Corbett, J. E., Oriet, C., \& Rensink, R. A. (2006). The rapid extraction of numeric meaning. Vision Research, 46(10), 1559-1573.

Dakin, S. C., \& Watt, R. J. (1997). The computation of orientation statistics from visual texture. Vision Research, 37, 3181-3192. https:// doi.org/10.1016/S0042-6989(97)00133-8

Dakin, S. C., Mareschal, I., \& Bex, P. J. (2005). Local and global limitations on direction integration assessed using equivalent noise analysis. Vision Research, 45(24), 3027-3049.

Dakin, S. C., Bex, P. J., Cass, J. R., \& Watt, R. J. (2009). Dissociable effects of attention and crowding on orientation averaging. Journal of Vision, 9(11), 28-28.

De Fockert, J. W., \& Marchant, A. P. (2008). Attention modulates set representation by statistical properties. Perception \& Psychophysics, 70(5), 789-794.

Faulkenberry, T. J., Cruise, A., Lavro, D., \& Shaki, S. (2016). Response trajectories capture the continuous dynamics of the size congruity effect. Acta Psychologica, 163, 114-123.

Faulkenberry, T. J., Ly, A., \& Wagenmakers, E. J. (2020). Bayesian inference in numerical cognition: A tutorial using JASP.

Haberman, J., \& Whitney, D. (2007). Rapid extraction of mean emotion and gender from sets of faces. Current biology, 17(17), R751-R753.

Haberman, J., \& Whitney, D. (2009). Seeing the mean: ensemble coding for sets of faces. Journal of Experimental Psychology: Human Perception and Performance, 35(3), 718.

Haberman, J., Harp, T., \& Whitney, D. (2009). Averaging facial expression over time. Journal of Visualization 9(11), 1-13.

Haberman, J., \& Whitney, D. (2010). The visual system discounts emotional deviants when extracting average expression. Attention, Perception, \& Psychophysics, 72(7), 1825-1838.

Haberman, J., Brady, T. F., \& Alvarez, G. A. (2015). Individual differences in ensemble perception reveal multiple, independent levels of ensemble representation. Journal of Experimental Psychology: General, 144(2), 432.

Hansen, T., Olkkonen, M., Walter, S., \& Gegenfurtner, K. R. (2006). Memory modulates color appearance. Nature Neuroscience, $9(11)$, $1367-1368$.

Huang, L. (2015). Statistical properties demand as much attention as object features. PLoS One, 10(8), e0131191.

Im, H. Y., \& Halberda, J. (2013). The effects of sampling and internal noise on the representation of ensemble average size. Attention, Perception, \& Psychophysics, 75(2), 278-286.

JASP Team (2020). JASP (Version 0.12.1.0)[Computer software]. Retrieved from https://jasp-stats.org/.
Kanaya, S., Hayashi, M. J., \& Whitney, D. (2018). Exaggerated groups: Amplification in ensemble coding of temporal and spatial features. Proceedings of the Royal Society B: Biological Sciences, 285(1879), 20172770.

Lee, K. R., Sobel, K. V., York, A. K., \& Puri, A. M. (2018). Dissociating parallel and serial processing of numerical value. Journal of Numerical Cognition, 4(2), 360-379.

Lupyan, G. (2008). The conceptual grouping effect: Categories matter (and named categories matter more). Cognition, 108(2), 566-577.

Lupyan, G., Thompson-Schill, S. L., \& Swingley, D. (2010). Conceptual penetration of visual processing. Psychological Science, 21(5), 682691.

Marchant, A. P., Simons, D. J., \& de Fockert, J. W. (2013). Ensemble representations: Effects of set size and item heterogeneity on average size perception. Acta Psychologica, 142(2), 245-250.

Marsman, M., \& Wagenmakers, E. J. (2017). Bayesian benefits with JASP. European Journal of Developmental Psychology, 14(5), $545-555$.

McClelland, T., \& Bayne, T. (2016). Ensemble coding and two conceptions of perceptual sparsity. Trends in Cognitive Sciences, 20(9), 641-642.

Oriet, C., \& Corbett, J. (2008). Evidence for rapid extraction of average size in RSVP displays of circles [Abstract]. Journal of Vision, 8(6), Article 13. https://doi.org/10.1167/8.6.13

Parkes, L., Lund, J., Angelucci, A., Solomon, J. A., \& Morgan, M. (2001). Compulsory averaging of crowded orientation signals in human vision. Nature Neuroscience, 4, 739-744. https://doi.org/ $10.1038 / 89532$

Puri, A. M., \& Wojciulik, E. (2008). Expectation both helps and hinders object perception. Vision Research, 48(4), 589-597.

Robitaille, N., \& Harris, I. M. (2011). When more is less: Extraction of summary statistics benefits from larger sets. Journal of Vision, 11(12), 18-18.

Santens, S., \& Verguts, T. (2011). The size congruity effect: Is bigger always more?. Cognition, 118(1), 94-110.

Schwarz, W., \& Heinze, H. J. (1998). On the interaction of numerical and size information in digit comparison: A behavioral and event-related potential study. Neuropsychologia, 36(11), 1167-1179.

Smith, A. R., \& Price, P. C. (2010). Sample size bias in the estimation of means. Psychonomic Bulletin \& Review, 17(4), 499-503.

Sobel, K. V., Puri, A. M., \& Hogan, J. (2015). Target grouping in visual search for multiple digits. Attention, Perception, \& Psychophysics, 77(1), 67-77.

Sobel, K. V., Puri, A. M., Faulkenberry, T. J., \& Dague, T. D. (2017). Visual search for conjunctions of physical and numerical size shows that they are processed independently. Journal of Experimental Psychology: Human Perception and Performance, 43(3), 444.

Solomon, J. A. (2010). Visual discrimination of orientation statistics in crowded and uncrowded arrays. Journal of Vision, 10(14), 19-19.

Sweeny, T. D., Haroz, S., \& Whitney, D. (2013). Perceiving group behavior: Sensitive ensemble coding mechanisms for biological motion of human crowds. Journal of Experimental Psychology: Human Perception and Performance, 39(2), 329.

Sweeny, T. D., \& Whitney, D. (2014). Perceiving crowd attention: Ensemble perception of a crowd's gaze. Psychological Science, 25(10), 1903-1913.

Tanaka, J. W., \& Farah, M. J. (2003). The holistic representation of faces. Perception of faces, objects, and scenes: Analytic and holistic processes (pp. 53-74). New York, NY: Oxford University Press.

Utochkin, I. S., \& Tiurina, N. A. (2014). Parallel averaging of size is possible but range-limited: A reply to Marchant, Simons, and De Fockert. Acta Psychologica, 146, 7-18. 
Van Opstal, F., de Lange, F. P., \& Dehaene, S. (2011). Rapid parallel semantic processing of numbers without awareness. Cognition, 120(1), 136-147.

Walsh, V. (2003). A theory of magnitude: common cortical metrics of time, space and quantity. Trends in Cognitive Sciences, 7(11), 483488.

Watamaniuk, S. N. J., Sekuler, R., \& Williams, D. W. (1989). Direction perception in complex dynamic displays: The integration of direction information. Vision Research, 29, 47-59. https://doi.org/10. 1016/0042-6989(89)90173-9

Whitney, D., \& Yamanashi Leib, A. (2018). Ensemble perception. Annual review of psychology, 69, 105-129.

Williams, D. W., \& Sekuler, R. (1984). Coherent global motion percepts from stochastic local motions. Vision Research, 24, 55-62.
Wolfe, B. A., Kosovicheva, A. A., Leib, A. Y., Wood, K., \& Whitney, D. (2015). Foveal input is not required for perception of crowd facial expression. Journal of Vision, 15(4), 11-11.

Yamanashi Leib, A., Fischer, J., Liu, Y., Qiu, S., Robertson, L., \& Whitney, D. (2014). Ensemble crowd perception: A viewpoint-invariant mechanism to represent average crowd identity. Journal of Vision, 14(8), 26-26.

Yamanashi Leib, A. Y., Kosovicheva, A., \& Whitney, D. (2016). Fast ensemble representations for abstract visual impressions. Nature Communications, 7(1), 1-10.

Publisher's note Springer Nature remains neutral with regard to jurisdictional claims in published maps and institutional affiliations. 